\title{
MEASUREMENT OF VISCOSITY IN SMALL VOLUMES OF FLUIDS BY TUNING FORK OSCILLATORS
}

\author{
Hana DIVIŠOVÁ, Jan LANG, Miloš ROTTER, and David SCHMORANZER *
}

\begin{abstract}
We report on the construction and performance of a home-built viscometer employing a quartz tuning fork resonator as the sensing element. The resulting device allows measuring the viscosity of fluids in volumes as low as $300 \mu \mathrm{l}$, while controlling the temperature between $-120^{\circ} \mathrm{C}$ and $150^{\circ} \mathrm{C}$. For non-conducting fluids, bare tuning forks can be used, reaching the accuracy of $1 \%$ or better in the whole temperature range. For weakly conducting fluids such as aqueous solutions, an insulating coating of paint is applied, resulting in reduced sensitivity and accuracy. The capabilities and limitations of the viscometer are illustrated on the viscosity measurements of $n$-hexane (between $-90^{\circ} \mathrm{C}$ and $+50^{\circ} \mathrm{C}$ ) and water (between $10^{\circ} \mathrm{C}$ and $90^{\circ} \mathrm{C}$ ). Improvements regarding performance in conducting liquids and temperature stabilization are discussed.
\end{abstract}

\section{INTRODUCTION}

Today, many types of viscometers are known, widely used and commercially available. The most common representatives include U-tube viscometers and falling sphere viscometers, as well as various arrangements of capillary viscometers, rotary viscometers or vibrational ones [1, 2, 3, 4]. Each type is adapted to different measuring conditions, some are preferable for gases, others for liquids of varying magnitudes of viscosity, possibly including non-Newtonian liquids, gels, and suspensions. While these are highly successful in industry, in scientific research, they may be found inadequate for one reason. In the commercial viscometers, a large amount of the working fluid (at least tens of $\mathrm{ml}$, but ranging up to 1 litre) is typically required, while sometimes it is crucial to know the viscosity of samples prepared in $\mathrm{ml}$ or sub-ml amounts. For example, measurements of transport properties of molecular solutions by means of nuclear magnetic resonance (NMR) enhanced with pulsed magnetic field gradients (PFG NMR) provide information on processes such as oligomerization, molecular association and clustering. Knowledge of the viscosity of the studied solutions is, however, a necessary prerequisite in order to obtain accurate quantitative results. Liquid NMR samples are often prepared using isotopic enrichment and therefore do not typically exceed the volume of $1 \mathrm{ml}$. Thus, a method of accurate determination of viscosity of small amounts of potentially expensive samples in a large temperature range is needed urgently.

For this purpose, we have constructed a working prototype of a quartz tuning fork viscometer, which allows reliable measurements of viscosity in sub-ml volumes of fluids owing to the small dimensions and high sensitivity of the employed crystal oscillating at $2^{15} \mathrm{~Hz} \approx 32 \mathrm{kHz}$. The quartz tuning forks (see Fig. 1) are well-known devices used as frequency standards in industrial electronics such as digital watches, but have found many interesting uses in scientific research as well. They have many applications in atomic force microscopy $[5,6]$ and similar sensitive methods of studying material surfaces, as well as in fluid dynamics research. They have proved particularly useful in the studies of classical and quantum turbulence in cryogenic helium fluids ranging from the gaseous phases, through normal liquids to the superfluid phases of ${ }^{3} \mathrm{He}[7,8,9,10,11]$ and ${ }^{4} \mathrm{He}$, in which they can act as generators and detectors of turbulent flows [12,13], detectors of cavitation [14, 15], they can be used for studying acoustic properties of the helium isotopes or of their mixtures $[16,17,18]$, of or even as pressure-, thermo- or viscometers [19].

\footnotetext{
*Hana Divišová, divishan@seznam.cz; Jan Lang, Jan.Lang@mff.cuni.cz; Miloš Rotter, rotter@nbox.troja.mff.cuni.cz; David Schmoranzer, david.schmoranzer@gmail.com.

Authors' contact address: Katedra fyziky nízkých teplot, MFF UK, V Holešovičkách 2, 180 00, Praha 8
}

This is an Open Access article distributed under the terms of the Creative Commons Attribution License 2.0, which permits unrestricted use, distribution, and reproduction in any medium, provided the original work is properly cited. 

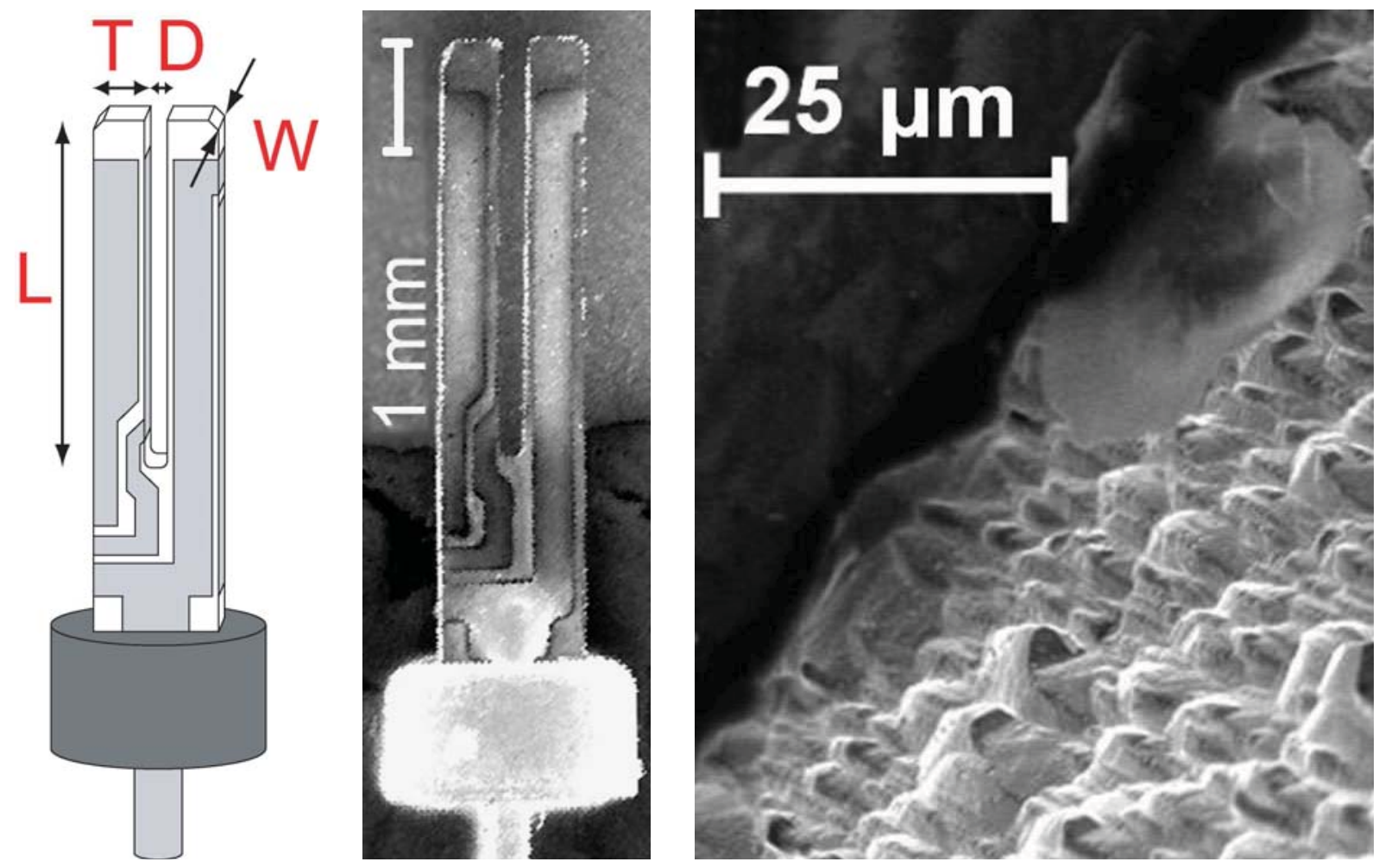

Figure 1: From left to right: (i) A sketch of the vibrating tuning fork including specifications of its dimensions. (ii) An electron micrograph of the $32 \mathrm{kHz}$ quartz tuning fork. (iii) A micrograph showing the typical surface roughness of the same commercially available tuning fork.

\section{TUNING Fork VISCOMETER - PRINCIPLE OF OPERATION}

The tuning fork consists of a piezoelectric quartz crystal with evaporation deposited (typically aluminium) electrodes and soldered or epoxy-glued leads. It is excited by AC voltage swept across its resonance frequency, while the measured electric current corresponds to the velocity of its oscillations [19]. When vibrating at low amplitudes (linear elasticity and laminar flow are required), the tuning fork is well-described by the Euler-Bernoulli beam theory [20], which can be conveniently simplified to a 1D linear harmonic oscillator by considering only the tips of the oscillating prongs. Its frequency characteristics can thus be described by a Lorentzian function characterised by resonance frequency, resonant linewidth and amplitude.

In vacuum, the resonance frequency of the tuning fork corresponds to its effective mass and stiffness parameters, while its linewidth is given by the very low intrinsic dissipation inside the quartz crystal and the electrodes. When submerged into a viscous fluid, the effective mass increases due to its hydrodynamical enhancement resulting in a lower resonance frequency, while viscosity will introduce additional dissipation of energy, which will lead to an increased linewidth. The full mechanical, electrical and hydrodynamical model of the tuning fork is presented in detail in Ref. [19], here we will only show the equations linking its resonance frequency, $f_{0}$, and its linewidth, $\Delta f$, to the physical properties of the medium in which it is immersed:

$$
\begin{aligned}
\left(\frac{f_{\text {Ovac }}}{f_{0}}\right)^{2} & =1+\frac{\rho}{\rho_{\mathrm{q}}}\left(\beta+B \frac{S}{V} \sqrt{\frac{\eta}{\pi \rho f_{0}}}\right), \\
\Delta f & =\Delta f_{\mathrm{vac}}+\frac{1}{2} \sqrt{\frac{\rho \eta f_{0}}{\pi}} \mathcal{C} S \frac{\left(f_{0} / f_{0 \mathrm{vac}}\right)^{2}}{m_{\mathrm{eff}}},
\end{aligned}
$$

where the index "vac" signifies values of the quantities as measured in vacuum, $\rho$ and $\rho_{q}$ are the densities of the surrounding fluid and of quartz, respectively, $\eta$ is the dynamic viscosity of the fluid. The quantities $V$ and $S$ are the volume and surface of a single prong of the tuning fork, and $m_{\mathrm{eff}}$ is its vacuum effective mass, which is exactly $1 / 4$ of its real mass according to the Euler-Bernoulli model, i.e., $V=T W L$, 
$S=2(T+W) L$ and $m_{\mathrm{eff}}=1 / 4 \rho_{q} V$. Finally $\beta, B$, and $C$ are geometry-dependent parameters, usually determined experimentally for each particular fork by fitting the data with the above equations.

From Eqs. 1 and 2 it follows that calibrated tuning forks can be used to measure the density and viscosity of a surrounding medium, provided that the medium is non-conducting and does not short-circuit the electrodes. Specifically in this work, we will use Eq. 2 to determine the fluid viscosity from the resonant characteristics of the tuning fork. It is therefore necessary to determine the parameter $C$, from a measurement in a fluid of known viscosity. It is also very useful to perform a self-calibration of the mechanical and electrical properties of the tuning fork [19], so that we know the velocity amplitudes during the measurement and can make sure to stay safely in laminar flow regime all the time.

For measurements of weakly conducting fluids (even such as de-ionized water), it is also necessary to provide an insulating layer to limit the parasitic currents through the conducting medium. Unfortunately, at the typical resonant frequency of the tuning fork $(32 \mathrm{kHz})$, sub-micronic layers of deposited plastic or ceramic materials, which do not reduce the quality of the oscillator too much, provide no significant impedance to the AC current, and in the end we had to use a thicker layer (tens of $\mu \mathrm{m}$ ) of heat-resistant paint to suppress the leakage currents efficiently. This, however, introduced significant additional damping, which also exhibited a strong temperature dependence and drifts, as is described in the section dealing with measurements in de-ionized water.

\section{CONSTRUCTION AND CALIBRATION OF THE VISCOMETER}

To build the viscometer prototype for measuring the samples previously studied by NMR, we re-used a NMR probe and a thermoregulation unit, that were parts of a commercial Bruker system [21] (see Fig. 2). The studied sample is held in a cuvette of $5 \mathrm{~mm}$ outer diameter and $\approx 3.8 \mathrm{~mm}$ inner diameter. The cuvette and its holder (NMR spinner) are located in the upper part of the NMR probe, which contains a glass dewar for thermal insulation, and has an installed heater/thermocouple pair for temperature control. The NMR probe is normally placed inside the bore of a superconducting NMR magnet, but in our case the magnet was not needed and the probe was kept open in room temperature surroundings. This would, of course, result in a reduced operational temperature range and for this reason we improved the insulation by coating the probe in alternating layers of plastic and aluminium foils.

The temperature of the sample was controlled using the above mentioned heater/thermocouple pair, which were connected to the Bruker thermoregulation unit that also allows controlling the flow of the thermalizing gas. In our case, the gas was either nitrogen entering at room temperature (for heating) or cold nitrogen vapours obtained by controlled evaporation of liquid nitrogen in a transport dewar. An additional platinum resistance thermometer was installed directly in the sample volume to determine the exact temperature in situ. We used the calibration of the thermometer provided by the manufacturer (Hayashi Denko) in the form of a fourth order polynomial expansion of $\mathrm{R}(\mathrm{T})$, with $0.1 \%$ nominal accuracy between $0{ }^{\circ} \mathrm{C}$ and $100^{\circ} \mathrm{C}$.

With this arrangement, several things had to be kept in mind. First, the tuning fork ( $\approx 1.5 \mathrm{~mm}$ width) had to be kept from touching or approaching the walls of the cuvette. For this purpose, two small stainless steel nuts were placed on the connected coaxial cables to provide centering. Second, with the thermometer below the tuning fork, we also had to prevent its 4 leads from touching the sensitive oscillator, which was achieved by sufficiently pre-twisting (and pre-stressing) them so that they followed the walls of the cuvette closely. Quantitatively this criterion can be expressed in such a way that there should be no solid objects or walls in several multiples of the viscous penetration depth $\delta=\sqrt{2 \nu / \omega}$, where $\nu$ is the kinematic viscosity, and $\omega$ is the angular frequency of oscillations. For the $32 \mathrm{kHz}$ tuning fork, in water at $273 \mathrm{~K}, \delta \approx 4.2 \mu \mathrm{m}$, and in n-hexane near its melting point, $\delta \approx 5.6 \mu \mathrm{m}$. We can therefore say, that if the prongs of the tuning fork are at least $0.1 \mathrm{~mm}$ away from other objects or walls, which is easily satisfied in the cuvette of $\approx 3.8 \mathrm{~mm}$ inner diameter, there should be no significant parasitic influences on the flow field around the oscillator, unless steady "streaming" flows occur.

An issue of more importance is the mutual vertical displacement of the thermometer and the tuning fork. This means that since most heating/cooling, as the case may be, occurs from the bottom of the cuvette (this is from where the thermalizing gas is flowing), it is possible that a vertical temperature gradient establishes in the sample. While the sample temperature is controlled "from the bottom", at its top level, the sample is exchanging heat with the air inside the cuvette, which is connected with the surrounding atmosphere through a tiny orifice in the cap provided for all the installed wires. Additionally, the sample also exchanges heat with the surrounding air through the weakly heat-conducting glass in the cuvette walls. For this reason, the measured values of viscosity may be slightly shifted on the temperature axis, 

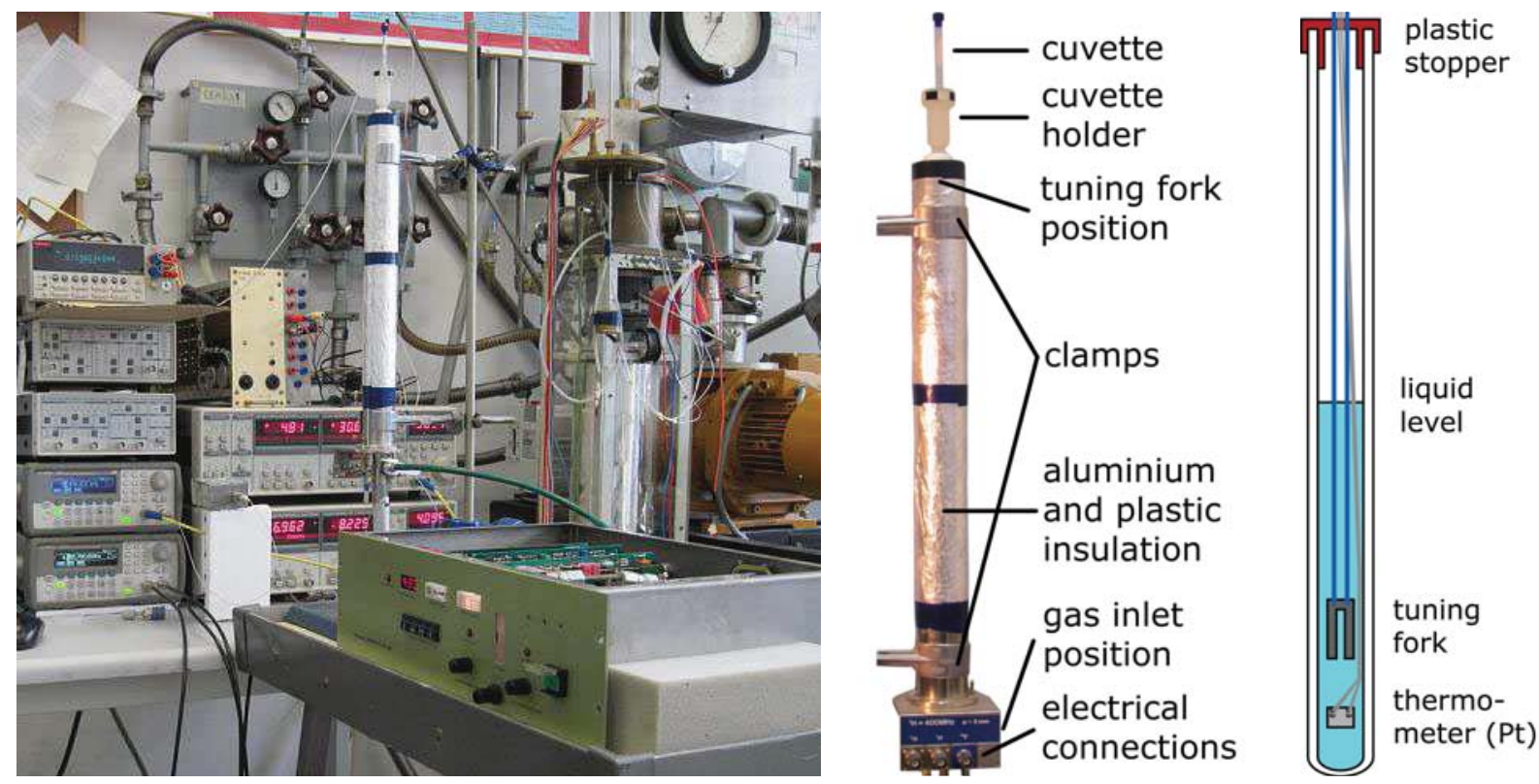

Figure 2: From left to right: (i) The entire experimental setup with the thermoregulation unit and the readout electronics (Agilent waveform generators A32200, and Stanford Research lock-in amplifiers SR-830. (ii) Schematics of the NMR probe. (iii) Sketch of the cuvette with the measured fluid, the tuning fork and a platinum resistance thermometer.

and the direction of this shift would depend on whether we are considering data above or below the room temperature of about $297 \mathrm{~K}$, and in case of rapid changes of temperature, also on the history of the sample.

Before the measurements began, it was necessary to calibrate the tuning fork. For this purpose, we used a closed volume of nitrogen gas at various pressures between 0 and 1 bar. We obtained the pressure dependence of the resonant linewidth of the tuning fork and using the known viscosity $\left(\eta=1.7796 \times 10^{-5} \mathrm{~Pa} \cdot \mathrm{s}\right)$ and other material properties of the nitrogen gas, it was possible to determine parameter $C$ in Eq. 2 as $C=0.625$. As the statistical uncertainty of this parameter from the fit was unrealistically low, we estimate it to be at the level of $1 \%$ due to possible uncertainties in the other quantities entering Eq. 2, especially $S$ and $m_{\text {eff }}$.

The mechanical-electrical calibration of the tuning fork [19] was performed as well by measuring its I/V characteristics in vacuum, and it was found that for our tuning forks, we can indeed use a direct proportionality relation between the electric current, $I$, and the tip velocity $v$. The constant of proportionality, or "fork constant", $a$, was determined as $a \equiv I / v=2.79 \times 10^{-5} \mathrm{C} / \mathrm{m}$. Subsequently, we used such driving voltages at which the tip velocity was below $1 \mathrm{~mm} / \mathrm{s}$, and hence safely in laminar flow in all the measured fluids $(R e<1)$. This calibration also brings us information about the usability limits of the tuning fork viscometer, as it becomes evident that in highly viscous fluids such as glycerine, the amplitude of the oscillator would be too low to enable accurate measurements, and special adaptations, such as a robust impedance-matched step-up transformer would be required for the drive.

\section{Viscosity OF N-HEXANE}

After several preliminary tests, and calibration of the tuning fork, we proceeded to measure the viscosity of the non-conducting n-hexane (Sigma Aldritch, 99\% purity). Our sample was of sub-ml volume and reached the height of $4-5 \mathrm{~cm}$ in the cuvette of internal diameter $\approx 3.8 \mathrm{~mm}$. The tuning fork was placed in the lower half of the sample with the Pt thermometer still below it, as illustrated in Fig. 2(iii). The sample was heated or cooled through the glass cuvette, around which the thermalizing gas was flowing on the outside.

The experimental protocol consisted of stabilizing the sample at a given temperature (evaluated from continuous readings of the Pt thermometer), taking several (10-20) measurements of the full resonance curve of the tuning fork, and then proceeding to the next temperature setting. The resulting resonance 


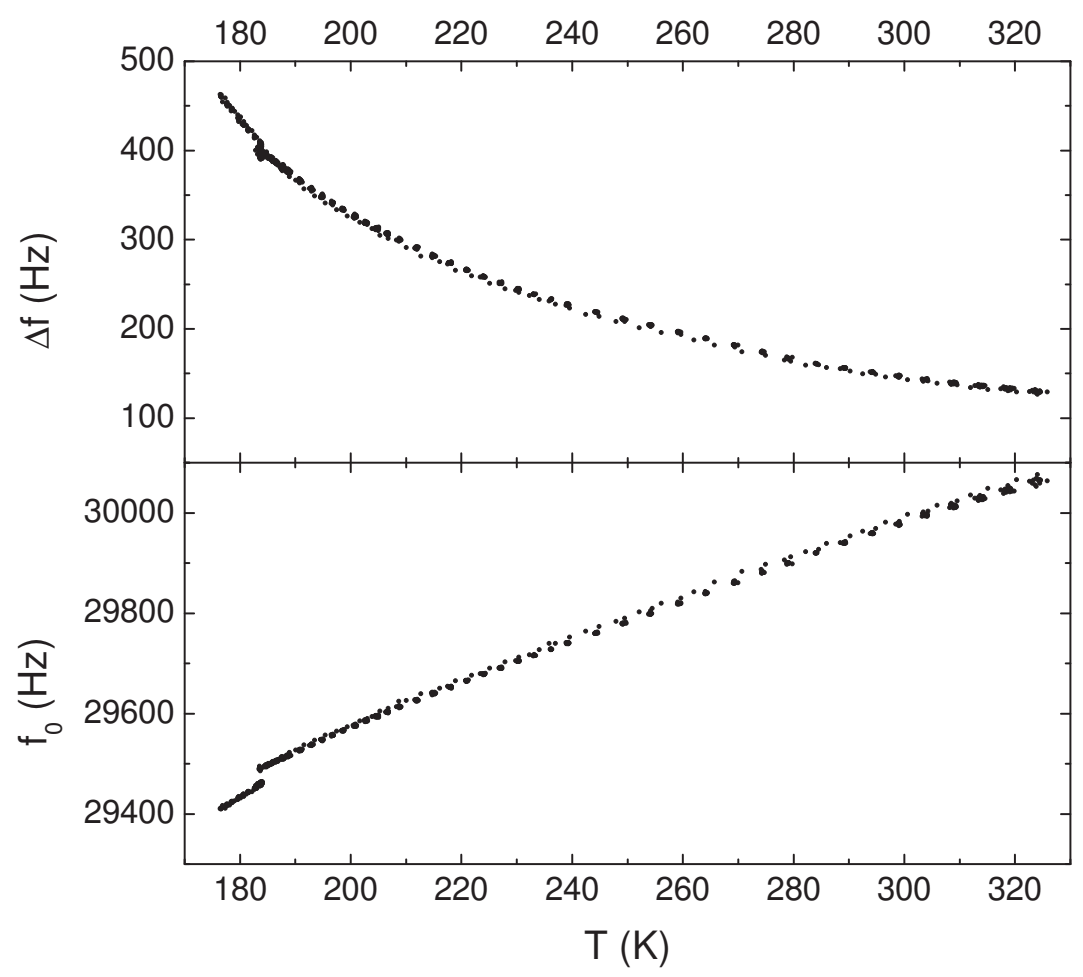

Figure 3: Resonant linewidths (top) and resonant frequencies (bottom) as measured by fitting the Lorentzian response of the tuning fork oscillator in $n$-hexane at various temperatures. The discontinuous data at the very lowest temperatures were measured separately after a warm-up to room temperature and a subsequent cooldown. The discontinuity is not physically significant here, it simply means, that between the two measurements, the experimental situation has changed appreciably, either due to the level drop of $n$-hexane in the cuvette upon heating (because of faster evaporation at high $\mathrm{T}$ ), or due to a nontrivial shift of the position of the oscillator to close vicinity of the cuvette's wall. These potentially problematic data points are excluded from the rest of the analysis.

characteristics are plotted in Fig. 3, including the data obtained while the temperature was still in transition from one value to another. These data were then filtered carefully to remove any bad measurements and the transitory periods. At each temperature, the obtained characteristics were processed statistically and the mean values were used to calculate the viscosity of $n$-hexane, which is presented in Fig. 4 and compared to reference values [22, 23].

From the presented figures it is evident that the tuning fork viscometer works very well in $n$-hexane and that it is capable of precise measurements of viscosity. The statistical uncertainties of the obtained averaged linewidths were less than $1 \mathrm{~Hz}$, which means that the largest uncertainty in the evaluated viscosity originates from the calibration of the tuning fork. We therefore claim that the viscometer is capable of attaining $1 \%$ accuracy in non-conducting liquids and gases, and that this could be further improved by more careful calibration procedures involving the use of several media of varied viscosities. Additional improvements should focus mainly on the design or adaptation of a more sophisticated thermostat, which would suppress the small temperature gradients in the sample and on measurements of temperature as close to the resonator as possible without disrupting the flow field, or possibly at multiple locations inside the sample simultaneously.

\section{VISCOSITY OF DE-IONIZED WATER}

In the next stage, we tested the capabilities of the viscometer in de-ionized water, as it is a very commonly used solvent in both organic and inorganic chemistry, and is often used for the biological samples studied by NMR methods. The sample we procured had a nominal resistivity of $\rho_{\mathrm{W}}=160 \mathrm{k} \Omega \mathrm{m}$, therefore, we could estimate the resistance, $R$, of water between the prongs of the tuning fork as 

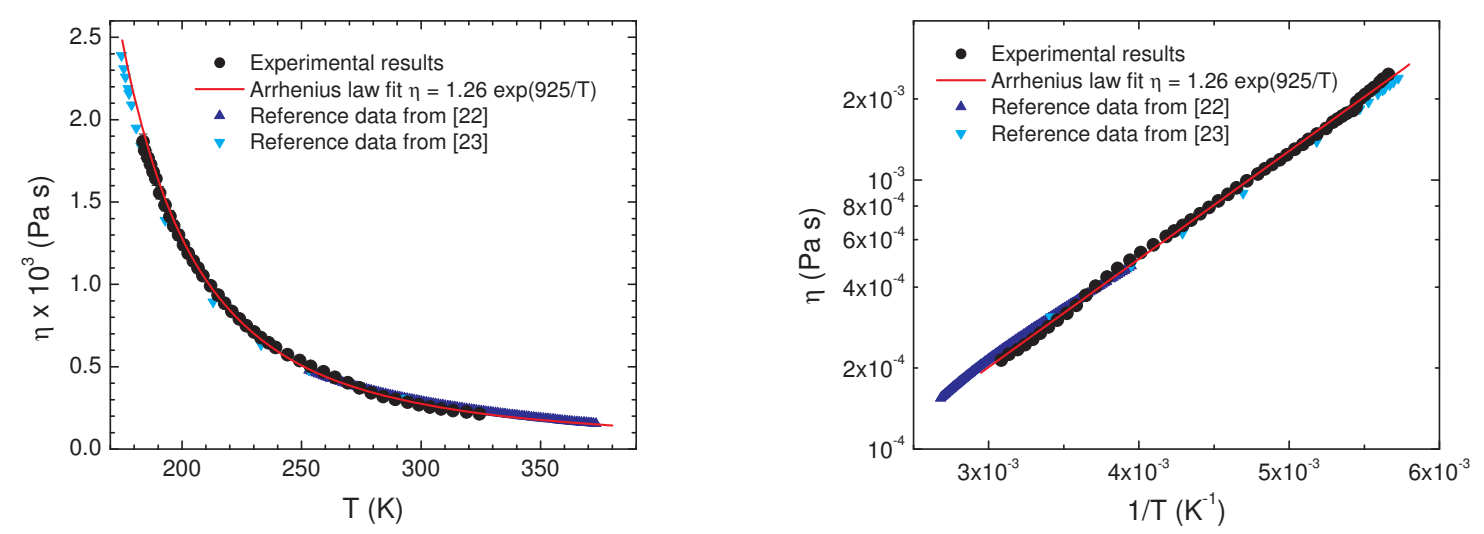

Figure 4: Temperature dependence of the viscosity of $n$-hexane measured by the tuning fork viscometer fitted with the Arrhenius law and compared to data from Refs. [22, 23] is shown in linear scale (left) and in semi-logarithmic scale with inverted temperature (right).

$R=\rho_{\mathrm{W}} D / L W \approx 10 \mathrm{M} \Omega$. The nominal impedance of the tuning fork at room temperature in vacuum is $40 \mathrm{k} \Omega$ at resonance, which corresponds to the vacuum linewidth measured as $\Delta f_{\mathrm{vac}}=0.5 \mathrm{~Hz}$. Away from the resonance, the impedance of the resonator gradually increases and diverges, and the measured signal will thus approach that of the conduction background. However, if we calculate the impedance for the highest obtained linewidths just above the melting point of water, it turns out to be about $30 \mathrm{M} \Omega$, and larger than the resistance of our sample of de-ionized water, albeit of the same order of magnitude.

This means, that a measurement of viscosity in this sample of water would be perhaps complicated, but still feasible. However, in practice, upon any manipulation in open atmosphere, the resistivity of de-ionized water tends to degrade significantly due to absorption of carbon dioxide and other gases, as well as due to other solid or liquid contaminants. For these reasons, without any insulation, the signal of the tuning fork is about $10^{3}$ times lower than the temperature-dependent and drifting conductivity background. Therefore, a practical measurement of viscosity of aqueous solutions is impossible without an insulating coating applied on the surface of the tuning fork and any unprotected parts of the leads.

In preparation of the experiment, we have tried several options for insulating the tuning fork, which would have minimum impact on its resonant characteristics and on its sensitivity. These included submicronic layers of deposited plastics ( $\mathrm{C}-\mathrm{H}$ networks or teflon) and ceramic materials, but unfortunately the methods employed have a prohibitive time cost if the layer thickness should be sufficient to really suppress the $32 \mathrm{kHz}$ AC current, which passes through thin insulating layers easily as a Maxwell current through a capacitor. In the end, the only possible solution was to coat the tuning fork with a spray paint [24], which creates layers of about 10-50 $\mu \mathrm{m}$ thickness. During the spray coating, the tuning fork was rotating along an external axis displaced by roughly $1 \mathrm{~cm}$ at several hundred $\mathrm{rpm}$, to provide as homogeneous coating as possible and also to remove excess paint by the centrifuge effect.

The resulting layer of insulating paint suppressed the background roughly one hundred times - to 10 times the level of the signal, but more importantly made it also significantly more stable, and thus allowed for the measurements to take place. Unfortunately, the paint also introduced significant excess damping on the tuning fork although it was carefully preselected and heat-treated according to specifications. This damping translates to linewidths of $10-100 \mathrm{~Hz}$ depending on temperature and had to be measured in advance and subtracted at each temperature from the results obtained in water in order to extract the viscosities presented in Fig. 5.

When compared with the measurements in $\mathrm{n}$-hexane the statistical scatter of the linewidths was much larger, which translated into the scatter of the viscosities as well. The values shown in Fig. 5 are each an average of 10-20 measurements, but still the uncertainties are reaching up to $10 \%$. The whole data series is also shifted by about $10 \%$ from the reference data, suggesting a systematic error likely resulting from an unexpected change in the damping contribution of the applied paint. The contribution of the paint 

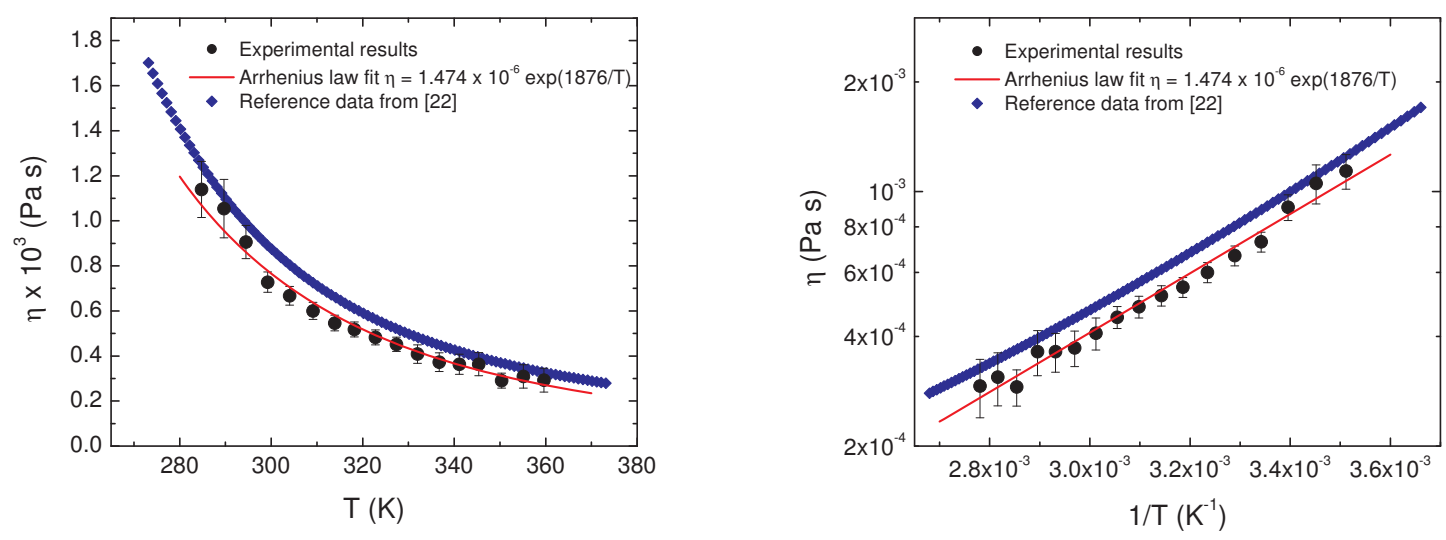

Figure 5: Temperature dependence of the viscosity of de-ionized water measured by the tuning fork viscometer fitted with the Arrhenius law and compared to data from Ref. [22] is shown in linear scale (left) and in semi-logarithmic scale with inverted temperature (right).

was measured separately as a function of temperature and subtracted, but it might have changed before or during the measurement in water, as the paint is already known to display irregular changes in its mechanical properties with thermal cycling and with time. Another possibility may be in the interaction of the paint with water, as it is known that some plastic materials tend to absorb water molecules into their structures, or release various chemicals into water upon prolonged exposure. In the end, we can say that for conducting polar liquids, the viscometer may have two significant sources of uncertainties - a systematic shift due to calibration of the paint contribution and a statistical one due to a strong and varying background. Both types of uncertainties amount to a relative error of up to $10 \%$.

The main improvements of the viscometer for use in conducting liquids are thus twofold. First, it would be highly advisable to find another coating material that is thermally, and chemically stable, does not degrade with time and can be applied in layers of the required thickness. Second, given a certain thickness of the insulating layer, it would be prudent to use a lower-frequency resonator, as the impedance of the layer (and the signal/background ratio) will increase in inverse proportion to the reduced frequency. The lowest usable frequency will be eventually limited by the dimensions of the oscillator, which must be kept sufficiently small to facilitate measurements in sub-ml volumes.

\section{Conclusions}

We have designed, constructed and tested a prototype of a tuning fork viscometer capable of measuring the viscosities of sub-ml samples in a wide temperature range. These properties grant the viscometer possible applications mainly in scientific research, where the studied fluids or mixtures are often available only in humble amounts. The accuracy of the viscometer was found to depend significantly on the type of fluid measured, especially on its conductivity, which determines whether the tuning forks can be used as they are, or whether an insulating coating is necessary. For non-conducting liquids with viscosities comparable to $\mathrm{n}$-hexane, the viscometer can be safely rated with an accuracy of $1 \%$, while for weakly conducting fluids of similar viscosities, the rating drops to $10 \%$ accuracy. Highly viscous fluids, as well as highly conducting ones, preclude the use of this type of viscometer unless additional modifications are performed. However, the viscometer can be expected to have a very good accuracy for fluids of lower dynamic viscosities (e.g., for gases), which typically cause difficulties with the traditional viscometers. In the future, the constructed instrument will be used at the Department of Low Temperature Physics of the Faculty of Mathematics and Physics in Prague for sensitive measurements of viscosity of low-volume samples, and will be gradually improved according to the suggestions mentioned in this report. 


\section{ACKNOWLEDGEMENTS}

The authors are grateful to Lenka Hanyková and Oleksandr Polonskyi for their help with the deposition of various insulating materials on the tuning forks and for interesting discussions on the related techniques and technologies. This research is supported by the Charles University Granting Agency under project GAUK 81410 and by the Czech Science Foundation GAČR 202/08/0276.

\section{REFERENCES}

[1] Rheotech Messtechnik a Divison of Bookfield. http://www.rheotec.de/de/?doc=products-lab-falling-kf10.

[2] Ubbelohde Capillary viscometer, http://capillaryviscometer.info/.

[3] Brookfield Viscometers, http://www.brookfieldengineering.com/products/viscometers/index.asp.

[4] Micro Motion 7827 Digital Viscosity Meter. http://www2.emersonprocess.com/en-US/brands/micromotion/density-viscosity-meters/.

[5] K. Karrai and R. D. Grober. Tip-sample distance control for near-field scanning optical microscopes. In M. A. Paesler and P. T. Moyer, editors, Near-Field Optics, volume 2535 of Proc. SPIE, pages 6981,1995

[6] R.D. Grober, J. Acimovic, J. Schuck, D. Hessman, P. J. Kindlemann, J. Hespanha, S. Morse, K. Karrai, I. Tiemann, and S. Manus. . Rev. Sci. Instrum., 71:2776, 2000.

[7] D. I. Bradley, M. J. Fear, S. N. Fisher, A. M. Guenault, R. P. Haley, C. R. Lawson, P. V. E. McClintock, G. R. Pickett, R. Schanen, V. Tsepelin, and L. A. Wheatland. Transition to Turbulence for a Quartz Tuning Fork in Superfluid He-4. J. Low Temp. Phys., 156(3-6):116-131, 2009.

[8] D. I. Bradley, P. Crookston, S. N. Fisher, A. Ganshin, A. M. Guenault, R. P. Haley, M. J. Jackson, G. R. Pickett, R. Schanen, and V. Tsepelin. The Damping of a Quartz Tuning Fork in Superfluid He-3-B at Low Temperatures. J. Low Temp. Phys., 157(5-6):476-501, 2009.

[9] M. Človečko, E. Gažo, M. Kupka, M. Skyba, and P. Skyba. High Quality Tuning Forks in Superfluid He-3-B Below 200 mu K. J. Low Temp. Phys., 162(5-6):669-677, 2011.

[10] D. I. Bradley, M. Človečko, E. Gažo, and P. Skyba. Probing Andreev reflection in superfluid He-3-B using a quartz tuning fork. J. Low Temp. Phys., 152(5-6):147-155, 2008.

[11] J. J. Hosio, V. B. Eltsov, R. de Graaf, M. Krusius, J. Mäkinen, and D. Schmoranzer. Propagation of thermal excitations in a cluster of vortices in superfluid 3He-B. arXiv:1103.2633v1 [cond-mat.other].

[12] M. Blažková, D. Schmoranzer, and L. Skrbek. Transition from laminar to turbulent drag in flow due to a vibrating quartz fork. Phys. Rev. E, 75(2, Part 2), 2007.

[13] D. Schmoranzer, M. Král'ová, V. Pilcová, W. F. Vinen, and L. Skrbek. Experiments relating to the flow induced by a vibrating quartz tuning fork and similar structures in a classical fluid. Phys. Rev. E, 81(6, Part 2), 2010.

[14] M. Blažková, T. V. Chagovets, M. Rotter, D. Schmoranzer, and L. Skrbek. Cavitation in liquid helium observed in a flow due to a vibrating quartz fork. J. Low Temp. Phys., 150(3-4):194-199, 2008. International Symposium on Quantum Fluids and Solids (QFS-2007), Kazan, Russia, Aug 01-06, 2007.

[15] M. Blažková, D. Schmoranzer, and L. Skrbek. On cavitation in liquid helium in a flow due to a vibrating quartz fork. Low Temp. Phys., 34(4-5):298-307, 2008. 
[16] D. Schmoranzer, M. La Mantia, G. Sheshin, I. Gritsenko, A. Zadorozhko, M. Rotter, and L. Skrbek. Acoustic Emission by Quartz Tuning Forks and Other Oscillating Structures in Cryogenic He-4 Fluids. J. Low Temp. Phys., 163(5-6):317-344, 2011.

[17] E. M. Pentti, J. T. Tuoriniemi, A. J. Salmela, and A. P. Sebedash. Quartz Tuning Fork in Helium. J. Low Temp. Phys., 150(3-4):555-560, 2008.

[18] E. M. Pentti, J. T. Tuoriniemi, A. J. Salmela, and A. P. Sebedash. Solubility of He-3 in He-4 at millikelvin Temperatures up to the Melting Pressure Measured by a Quartz Tuning Fork. Phys. Rev. $B, 78(6), 2008$.

[19] R. Blaauwgeers, M. Blažková, M. Človečko, V. B. Eltsov, R. de Graaf, J. Hosio, M. Krusius, D. Schmoranzer, W. Schoepe, L. Skrbek, P. Skyba, R. E. Solntsev, and D. E. Zmeev. Quartz tuning fork: Thermometer, pressure- and viscometer for helium liquids. J. Low Temp. Phys., 146(56):537-562, 2007.

[20] J. M. Gere and S. P. Timoshenko. Mechanics of Materials. PWS-KENT Publishing Company, Boston, MA, Third Edition edition, 1990.

[21] Bruker Variable Temperature Unit 1000 CTR, AM; Probe of a Bruker NMR spectrometer $\left({ }^{1} \mathrm{H}=400\right.$ $\mathrm{MHz}$, outer cuvetter diameter $5 \mathrm{~mm}$ ); Bruker $\mathrm{LN}_{2}$ evaporator.

[22] ASPEN database, http://www.aspentech.com/products/aspen-plus.aspx.

[23] E. B. Giller and H. G. Drickamer. Viscosity of normal paraffins near the freezing point. Industrial \& Engineering Chemistry, 41(9):2067-2069, 1949.

[24] Happy color, Smalto spray alte temperature (black), Sforza S.P.A., Italy. 